\title{
Warm-blood cardioplegic arrest induces selective mitochondrial translocation of protein kinase C $\epsilon$ followed by interaction with 6.1 inwardly rectifying potassium channel subunit in viable myocytes overexpressing urocortin
}

\author{
Carol Chen-Scarabelli, MSc, FAHA, ${ }^{\mathrm{a}, \mathrm{b}}$ Giuseppe Faggian, MD, ${ }^{\mathrm{b}}$ Zhaokan Yuan, PhD, ${ }^{\mathrm{c}}$ \\ Maddalena Tessari, MSc, ${ }^{\mathrm{b}}$ Alessio Rungatscher, MD,${ }^{\mathrm{b}}$ Justin Di Rezze, BSc, ${ }^{\mathrm{c}}$ Gabriele M. Scarabelli, BSc, ${ }^{\mathrm{c}}$ \\ Kadija Abounit, MSc, ${ }^{\mathrm{c}}$ Roy McCauley, $\mathrm{PhD},{ }^{\mathrm{c}}$ Louis Saravolatz, MD, ${ }^{\mathrm{c}}$ Alessandro Mazzucco, MD, ${ }^{\mathrm{b}}$ and \\ Tiziano M. Scarabelli, MD, PhD, FAHA ${ }^{\mathrm{c}}$
}

Objective: This study investigates the cardioprotective role and mechanism of action of urocortin in patients undergoing cardiac surgery, with respect to protein kinase $\mathrm{C} \epsilon$ expression, activation, and relocation.

Background: Cardioplegic arrest and subsequent reperfusion inevitably expose the heart to iatrogenic ischemia/ reperfusion injury. We previously reported that iatrogenic ischemia/reperfusion injury caused myocyte induction of urocortin, an endogenous cardioprotective peptide.

Methods: Two sequential biopsies were obtained from the right atrium of 25 patients undergoing coronary artery bypass grafting at the start of grafting (internal control) and 10 minutes after release of the aortic clamp.

\begin{abstract}
Results: In hearts exposed to iatrogenic ischemia/reperfusion injury, induction of urocortin was documented at both the mRNA $(255 \%$ of basic levels; $P<.05)$ and the protein (4-fold increase; $P<.01)$ levels. Iatrogenic ischemia/reperfusion injury also induced a selective increase of protein kinase $\mathrm{C} \epsilon$ mRNA $(225 \%$ of internal control; $P<.05)$ and a 2 -fold overexpression of total protein kinase $\mathrm{C} \epsilon(P<.05)$, which paralleled a 2.9-fold increase in protein kinase $\mathrm{C} \epsilon$ phosphorylation $(P<.01)$. Mitochondrial translocation of activated protein kinase $\mathrm{C} \epsilon$ was observed only in postcardioplegic samples, using both subcellular fractionation $(P<.05)$ and immunostaining techniques $(P<.05)$. Enhanced protein kinase $\mathrm{C} \epsilon /$ mitochondria colocalization was selectively observed in viable myocytes, showing concurrently positive staining for urocortin $(P<.05)$. Finally, co immunoprecipitation experiments documented an iatrogenic ischemia/reperfusion injury-enhanced physical interaction of phosphorylated protein kinase $\mathrm{C} \epsilon$ with the 6.1 inwardly rectifying potassium channel subunit of the $\mathrm{K}_{\mathrm{ATP}}$ channels $(P<.05)$.

Conclusion: After iatrogenic ischemia/reperfusion injury, urocortin expression in viable cells selectively colocalized with enhanced phosphorylation and mitochondrial relocation of protein kinase $\mathrm{C} \epsilon$, suggesting a cardioprotective role for endogenous urocortin. The physical interaction of activated protein kinase $\mathrm{C} \epsilon$ with 6.1 inwardly rectifying potassium channel, enhanced by cardioplegic arrest, may represent a conjectural mechanism of urocortin-mediated cardioprotection.
\end{abstract}

Cardioplegic arrest and subsequent reperfusion expose the human heart undergoing on-pump cardiac surgery to mild forms of ischemia/reperfusion (I/R) injury. These controlled forms of surgical cardiac arrest are likely to represent, together with the short coronary occlusions induced by balloon inflation during percutaneous transluminal coronary

\footnotetext{
From the VA Ann Arbor Healthcare System, ${ }^{\text {a }}$ University of Michigan, Mich; Division of Cardiac Surgery, University of Verona, ${ }^{\mathrm{b}}$ Verona, Italy; and Center for Heart and Vessel Preclinical Studies, St John Hospital and Medical Center, Wayne State University Medical School, ${ }^{\mathrm{c}}$ Detroit, Mich.

There is no conflict of interest to be disclosed for any of the authors.

Received for publication June 6, 2008; revisions received Feb 23, 2009; accepted for publication March 28, 2009; available ahead of print June 24, 2009.

Address for reprints: Carol Chen-Scarabelli, MSc, FAHA, VA Ann Arbor Healthcare System, University of Michigan, 2215 Fuller Road (111A), Ann Arbor, MI 48105

(E-mail: cchensc@med.umich.edu).

J Thorac Cardiovasc Surg 2009;138:1213-21

$0022-5223 / \$ 36.00$

Copyright (c) 2009 by The American Association for Thoracic Surgery

doi:10.1016/j.jtcvs.2009.03.041
}

angioplasty, the most common expression of iatrogenic ischemia/reperfusion injury (iIRI). Several cardioplegic techniques, such as crystalloid, cold, and warm-blood cardioplegia, have been developed in the attempt to prevent or at least to reduce to a minimum this inevitable surgically related ischemic insult. ${ }^{1}$ Route and modality administration of cardioplegia (continuous vs intermittent; antegrade vs retrograde) have also been extensively modified and diversified to maximize the final degree of cardioprotection during cardiac surgery. Nonetheless, the protection afforded by the different techniques used so far was shown to be oftentimes inadequate, above all when case-related technical difficulties considerably prolong the overall time during which the heart is maintained on cardiopulmonary bypass. ${ }^{2-4}$

Apoptotic cell death has been extensively implicated in the pathogenesis of I/R injury, including the iatrogenic variants associated with on-pump cardiac surgery. DNA fragmentation has been detected by terminal deoxynucleotidyl 


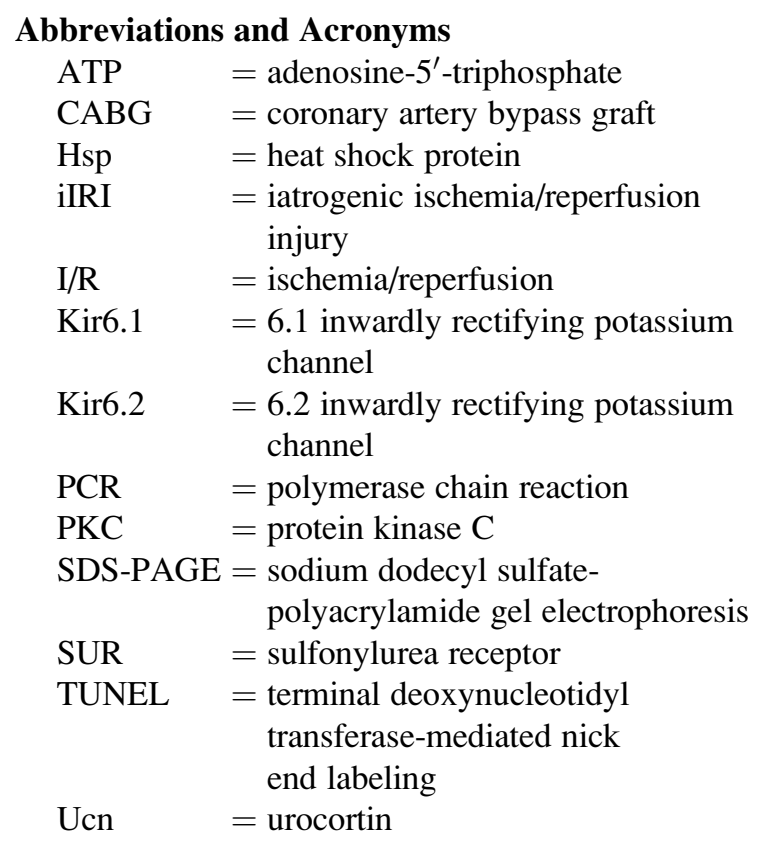

transferase-mediated nick end labeling (TUNEL) staining in atrial biopsies from patients protected by 3 different cold antegrade crystalloid cardioplegic solutions (St Thomas II [Köhler Chemie, Alsbach, Germany], Bretschneider [Köhler Chemie], or Hamburg [Fresenius AG, Bad Homburg, Germany]), as well as by cold-blood cardioplegia. ${ }^{5,6}$ The occurrence of apoptosis has also been evaluated and quantified by our group in the human heart from patients exposed to warmblood cardioplegic arrest and subsequent reperfusion. ${ }^{7}$ On the basis of our findings, warm-blood cardioplegia was indeed associated with myocyte apoptosis, but also with increased expression of urocortin (Ucn), a 40-amino acid cardioprotective peptide, member of the corticotropin releasing factorfamily. ${ }^{7}$ Myocytes overexpressing Ucn never exhibited TUNEL-positive staining, providing evidence that endogenous Ucn effectively protects those myocytes in which it is produced. ${ }^{7}$ In addition, viable myocytes overexpressing Ucn showed concurrent overexpression of the 6.1 inwardly rectifying potassium channel (Kir6.1) subunit of the adenosine- $5^{\prime}$-triphosphate (ATP)-sensitive inwardly rectifying potassium $\left(\mathrm{K}_{\mathrm{ATP}}\right)$ channel. These data are in line with previous experimental findings showing that administration of exogenous $U c n$ to a rat model of $I / R$ injury induced overexpression of the Kir6.1 cardiac $\mathrm{K}_{\mathrm{ATP}}$ channel subunit, without having any effect on the other 2 channel subunits, namely, 6.2 inwardly rectifying potassium channel (Kir6.2) and sulfonylurea receptor (SUR)-2. ${ }^{8}$ The $\mathrm{K}_{\mathrm{ATP}}$ channel is closed under normal conditions and functions as a sensitive sensor of the metabolic state of the cell. ${ }^{9}$ Opening of the cardiac $\mathrm{K}_{\mathrm{ATP}}$ channel, which is triggered by a decrease in intracellular ATP concentration, was shown to exert cardioprotective effects in numerous studies. ${ }^{10,11}$ The signaling pathways leading to activation and opening of the $\mathrm{K}_{\mathrm{ATP}}$ channels are still under investigation. Protein kinase $G$ was shown to transmit the cardioprotective signal to the $\mathrm{K}_{\mathrm{ATP}}$ channels through a protein kinase C (PKC)-dependent pathway, ${ }^{12}$ but it is still unclear how this signal is transmitted and which isoforms of PKC are involved in this process.

Recent works have increasingly identified in PKC $\epsilon$ the $\mathrm{PKC}$ isoform chiefly involved in mediating cardioprotection against $\mathrm{I} / \mathrm{R}$ injury and producing the phenomenon of ischemic preconditioning. ${ }^{13,14}$ Exogenous Ucn was shown to cause activation and mitochondrial translocation of PKC $\epsilon$, whereas pharmacologic inhibition of PKC $\epsilon$ and knockout of the PKC $\epsilon$ gene both resulted in abrogation of Ucn's cardioprotective effects, ${ }^{15}$ suggesting that Ucn-mediated cardioprotection is dependent on PKC $\epsilon$ activation.

Despite the redundancy of experimental data, the existence of a Ucn-activated cardioprotective pathway acting on the myocyte mitochondrion was never investigated in the human heart.

Thus, this study aimed to investigate the following in the human heart exposed to warm-blood cardioplegic arrest and subsequent reperfusion: (1) the changes in expression, activation, and subcellular localization of PKC $\epsilon$ induced by endogenous Ucn; (2) the existence of specific mitochondrial partners interacting with $\mathrm{PKC} \epsilon$; and (3) the role played by the above changes in Ucn-mediated cardioprotection.

\section{MATERIALS AND METHODS \\ Patient Population}

The study was approved by the institutional ethics committee of the University of Verona, and all patients selected gave informed consent before enrollment. Twenty-five patients ( 11 female and 14 male) admitted for elective on-pump coronary artery bypass graft (CABG) surgery, were recruited. All patients had multiple-vessel coronary disease with symptoms of class II/ III, according to the Canadian Cardiovascular Society Angina Classification. Exclusion criteria were (1) symptoms of angina pectoris or heart failure during the week preceding the operation; (2) reduced left ventricular ejection fraction $(<40 \%)$; (3) enlargement or hypertrophy of ventricular or atrial cardiac chambers; d) concomitant valvular disease; (4) arrhythmias; (5) diabetes and other metabolic disorders; and (6) concomitant liver, pulmonary, or kidney diseases. The intentional enrollment of subjects who were otherwise healthy, unlike the patient population typically undergoing CABG surgery, although representing a limitation of our study, also has the merit of restricting the degree of interference that comorbidities might have exerted. In addition, another study comparing the effects of cardioplegic arrest in diabetic versus nondiabetic patients is currently being carried out by our group. All patients were receiving calcium channel blockers and $\beta$-blockers, which were discontinued, together with all other medications, 2 days before surgery.

\section{Surgical Procedure}

Cardiopulmonary bypass was conducted with nonpulsatile flow at a rate of $2.4 \mathrm{~L} / \mathrm{min} / \mathrm{m}^{2}$. After aortic crossclamping, intermittent antegrade and retrograde warm-blood cardioplegia was initiated through the infusion of whole blood plus $20 \mathrm{mEq} / \mathrm{L}$ of $\mathrm{K}^{+}$. The cardioplegic solution was injected at $37^{\circ} \mathrm{C}$ for 2 minutes into the aortic root (flow rate, $300 \mathrm{~mL} / \mathrm{min}$ ) and for 
an additional 2 minutes into the coronary sinus at approximately $150 \mathrm{~mL} /$ $\min (\sim 40 \mathrm{~mm} \mathrm{Hg})$. This dose of cardioplegic solution was infused every 15 to 20 minutes via antegrade and retrograde injection. After completion of the distal anastomoses, the aortic crossclamp was removed and the construction of the proximal anastomoses was begun. At the end of the grafting procedure, protamine ( $3 \mu \mathrm{g} / \mathrm{kg}$ ) was injected to reverse the effect of heparin. Inotropic drugs were not used.

\section{Tissue Sampling and Preparation}

Our histologic and molecular evaluations have been carried out in bioptic tissues from the right atrium, whose changes during cardioplegic arrest and subsequent may not fully reflect those occurring in ventricular myocytes.

Two sequential biopsies were obtained from a virgin site in the right atrium by 2 highly experienced cardiac surgeons (G.F. and A.M.). The first biopsy was taken as a control sample before starting cardiopulmonary bypass. The second was obtained as close as possible to the site of the previous sampling approximately 10 minutes after the release of the aortic crossclamp. There were no clinical complications related to the sampling procedure. Immediately after collection, each atrial biopsy was cut into 3 parts. The first portion was fixed in $4 \%$ formaldehyde, stored at $4{ }^{\circ} \mathrm{C}$ for a maximum of 48 hours, subsequently wax embedded, and finally used for immunofluorescent staining. The second portion was stored at $4{ }^{\circ} \mathrm{C}$ in RNAlater Solution (Ambion, Austin, Tex) and subsequently used for RNA isolation. The third portion, immediately after collection, was parceled out into 3 fragments, 2 of which were collected into separate cryovials, snapfrozen in liquid nitrogen, stored at $-80^{\circ} \mathrm{C}$, and finally used for protein isolation from total tissue extracts. The third and final fragment was collected into ice-cold phosphate-buffered saline and immediately used for isolation of mitochondrial and cytoplasmic fractions (see below). To obtain adequate amounts of proteins, pre- and postcardioplegic samples collected from batches of 3 different patients (with the exception of 1 batch composed of 4) were combined and processed together.

\section{RNA Isolation and Quantitative Polymerase Chain Reaction}

Total RNA was isolated from cardiac samples by using an RNA isolator solution TRIzol (Gibco-BRL, Sussex, United Kingdom), treated with DNAse (Promega, Madison, WI) and subsequently reversed transcribed with Superscript II reverse transcriptase (Gibco BRL) and oligodT (18mer) primer. ${ }^{16}$ Real-time, 1-step polymerase chain reaction (PCR) for specific mRNA was performed using the QuantiTect SYBR Green PCR Kit (Qiagen, Venlo, The Netherlands) and LightCycler 1.5 Instrument (Roche Diagnostics, Indianapolis, IN), according to the manufacturer's instructions. The following specific primers were used to quantitate by qPCR the transcript levels of the genes of interest. Ucn primer sequences were $5^{\prime}$-GCT TGCTGGTGAAAAGGACC- $3^{\prime}$ (sense) and $5^{\prime}$-CTTGCCCACCGAGTCG AAT- $3^{\prime}$ (antisense). PKC $\epsilon$ primer sequences were $5^{\prime}$-ACCAAGCAGAAG ACCAACAG- $3^{\prime}$ (sense) and $5^{\prime}$-TTCCTATGACACCCCAGATG- $3^{\prime}$ (antisense).

\section{Immunoblotting Material and Approach}

Types and sources of the antibody used for the immunoblotting procedure are as follows: The antibody against actin, heat shock protein (Hsp)60, total PKC $\epsilon$, Kir6.1 (H80), Kir6.2 (H55), and SUR-2 (H80) were obtained from Santa Cruz Biotechnology (Santa Cruz, Calif). The antiphospho-PKC $\epsilon$ (Ser729) rabbit polyclonal antibody was purchased from Upstate Biotechnology (Lake Placid, NY). All secondary antibodies were conjugated to horseradish peroxidase and the ensuing immunoreactive bands were developed using a Western Lightning Chemiluminescence kit (PerkinElmer Life Science, Boston, Mass). The nitrocellulose membrane used for Western blotting was obtained from Bio-Rad Laboratories (Hercules, Calif). Immunoblots were digitalized and quantified using ImageJ software (National Institutes of Health, Bethesda, Md).

\section{Western Blotting}

Pre- and postcardioplegic cardiac specimens were homogenized in lysis buffer (100 mmol/L NaCl, 0.1\% Triton X-100, 50 mmol/L Tris; pH: 8.3) loaded on $8 \%$ sodium dodecyl sulfate (SDS)-polyacrylamide gel, separated by gel electrophoresis, transferred onto a nitrocellulose membrane, and probed with relevant primary antibody. For the specific assessment of Ucn protein, whose molecular weight is 4693.52 dalton, we used a tricine SDS-polyacrylamide gel electrophoresis (PAGE) system, composed of $16 \% \mathrm{~T}$ and $6 \% \mathrm{C}$, where $\mathrm{T}$ indicates the total percentage concentration of both monomers (acrylamide and the crosslinker bisacrylamide) and $\mathrm{C}$ indicates the percentage concentration of the crosslinker relative to the total concentration. ${ }^{17}$ The SDS-PAGE system was enriched with $6 \mathrm{~mol} / \mathrm{L}$ urea to reduce the electrophoretic mobility of small proteins, with subsequent enhanced resolution of peptides having a molecular weight less than $5 \mathrm{kDa}$.

\section{Mitochondrial and Cytoplasmic Protein Fractionation, Characterization, and Assessment}

Mitochondrial and cytosolic fractions were prepared using a commercially available kit (Mitochondria Isolation Kit for Tissue; Pierce Protein Research Products, Thermo Fisher Scientific, Rockford, Ill), according to the manufacturer's instructions. Equal amounts $(20 \mu \mathrm{g})$ of cytoplasmic and mitochondrial proteins were separated using SDS-PAGE, as previously described, ${ }^{18,19}$ and subsequently probed with antibody against total and phosphorylated PKC $\epsilon$, Kir6.1, Kir6.2, and SUR-2. Hsp60 and actin antibody were used to probe for mitochondrial and cytoplasmic fractions, respectively.

\section{Co-immunoprecipitation}

After homogenization of the myocardial tissues in lysis buffer, phosphorylated PKC $\epsilon$ was enriched by immunoprecipitation overnight at $4{ }^{\circ} \mathrm{C}$ and subsequently immunoblotted with Kir6.1, Kir6.2, or SUR-2 antibody, as previously described. ${ }^{20}$ Western blotting was performed following standard protocols with equal amounts of protein loaded for each sample.

\section{Immunocytochemistry Assessment}

Serial 5- $\mu \mathrm{m}$ myocardial sections were stained with antibody against phosphorylated PKC $\epsilon$ and Hsp60 (used as a specific marker of mitochondria), and subsequently counterstained with TOPRO-3. In another set of staining reactions, adjacent $5-\mu \mathrm{m}$ myocardial sections were serially cut from the same wax blocks, stained with TUNEL reagents or an antibody against Ucn, counterstained with propidium iodide, and finally incubated with suitable secondary antibodies (DakoCytomation, Glostrup, Denmark), as previously described. ${ }^{7,19,21}$ To assess the mitochondrial relocation of activated PKC $\epsilon$, the phosphorylated active form of PKC $\epsilon$ and the mitochondria were labeled with specific primary antibody and visualized by incubation with secondary antibody conjugated with fluorescein and rhodamine, respectively. The percentage of colocalization of phosphorylated PKC $\epsilon$ and mitochondria was digitally quantified by means of image analyzer software in myocardial sections from samples collected before and after cardioplegic arrest. Sections were analyzed by a confocal microscopist (T.M.S.) who was blinded to the origin and sequence of the specimens. Data were expressed as the means of 12 to 15 high-power fields ( \pm standard deviation).

\section{Statistical Analysis}

Significance was evaluated using the 2-tailed analysis of variance.

\section{RESULTS}

\section{Patient Characteristics}

Operative details and clinical characteristics of the enrolled patients are summarized in Table 1 . All subjects were white and aged between 51 and 78 years (mean, $63 \pm 5$ years) 
TABLE 1. Operative details and clinical characteristics of enrolled patients

\begin{tabular}{lc}
\hline \multicolumn{1}{c}{ Variables } \\
\hline No. & 25 \\
Female & 11 \\
Male & 14 \\
Age (y) & $63 \pm 5$ \\
Aortic crossclamp (mean \pm SD, min) & $48 \pm 8$ \\
Range (min) & $42-56$ \\
Grafts & $3.6 \pm 0.5$ \\
LV ejection fraction (\% pre-CAGB) & $54 \pm 1$ \\
LV ejection fraction (\% post-CABG) & $52 \pm 3$ \\
\hline$S D$, Standard deviation; $L V$, left ventricular; $C A B G$, coronary artery bypass grafting. \\
Data are expressed as mean \pm SD.
\end{tabular}

with multiple-vessel coronary disease and symptoms of class II/III, according to the Canadian Cardiovascular Society Angina Classification. As previously stated, the intentional recruitment of patients without significant comorbidities, although introducing a limitation in our study, has the advantage of minimizing the concurrence of unknown confounding factors intrinsic to diverse pathologic conditions. All patients electively underwent multiple CABGs with internal thoracic arteries and saphenous veins. Mean values of pre- and postoperative ejection fraction were $54 \pm 1$ and $52 \pm 3$, respectively. In no case was catecholamine support required. Aortic crossclamping time ranged from 42 to 56 minutes (mean, $48 \pm$ 8 minutes), and the mean number of grafts was $3.6 \pm 0.5$. All data are presented as mean \pm standard deviation. None of the enrolled patients had complications during the surgical procedure or postsurgical follow-up.

\section{Enhanced Expression of Urocortin mRNA and Protein After Cardioplegic Arrest}

To assess changes in gene expression of Ucn, we prepared samples of mRNA from the myocardial biopsies and quantified the change in Ucn mRNA by real-time PCR. The results of this experiment, shown in Figure 1, A, documented a significant increase in Ucn RNA levels after cardioplegic arrest $(255 \%$ of basic levels, $P<.05)$.

To extend this observation further, protein extracts were also prepared from the cardiac biopsies and probed with a specific antibody to Ucn. As shown in Figure 1, B, a 4-fold increase was observed in the protein abundance of Ucn in the atrial specimens exposed to cardioplegic arrest $(P<.05)$.

Thus, the results of 2 independent assays, involving either mRNA quantification by real-time PCR or protein assessment by Western blotting, confirmed a significant increase in Ucn mRNA and protein levels in the postcardioplegic atrial biopsies, compared with the precardioplegic internal controls.

\section{Induction and Activation of Protein Kinase $\mathrm{C} \epsilon$ in Postcardioplegic Atrial Biopsies}

We then investigated whether the enhanced expression of Ucn induced by cardioplegic arrest was associated with
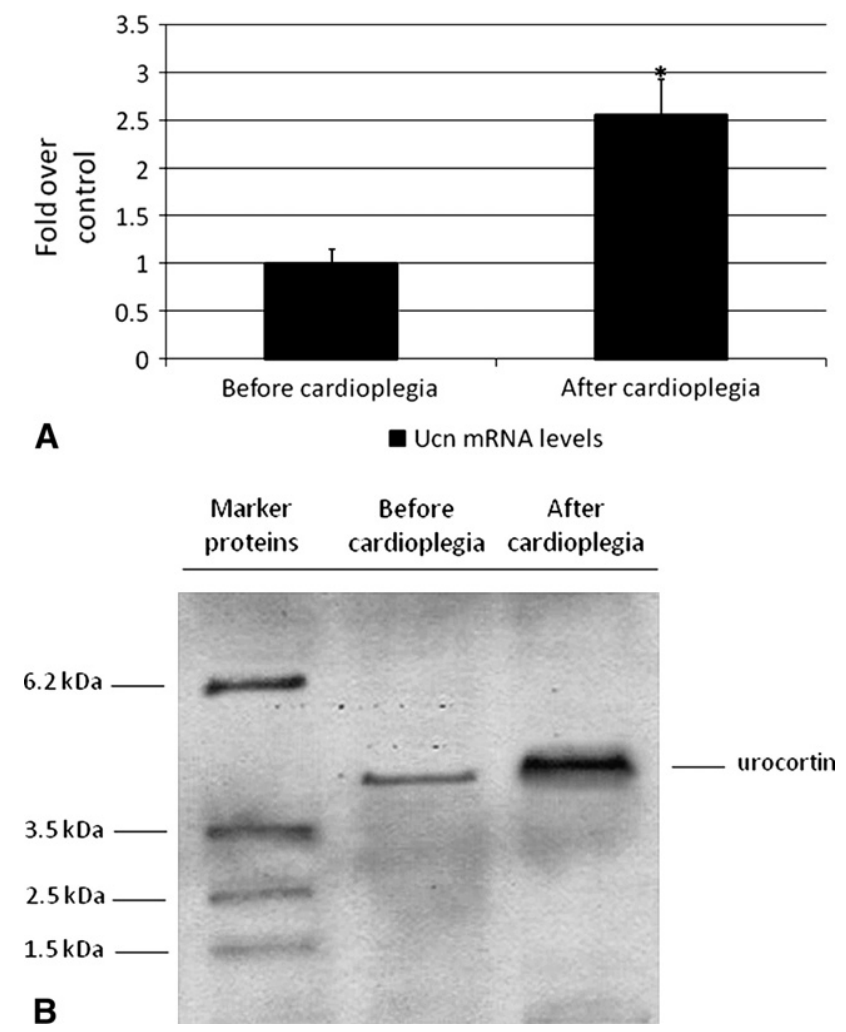

FIGURE 1. A, Quantitative PCR of Ucn mRNA levels in cardiac samples collected before and after cardioplegic arrest. $* P<.05$ versus corresponding precardioplegic internal controls. B, Expression of the Ucn protein, as determined by Western blotting of extracts from pre- and postcardioplegic atrial tissues. Protein extracts were probed with a specific antibody to Ucn in a 16\% T, 6\% C Tricine-SDS-PAGE system, complemented with $6 \mathrm{~mol} / \mathrm{L}$ urea. Marker proteins with the indicated molecular masses were also used. $U c n$, Urocortin.

changes in gene expression of PKC $\epsilon$. To this end, the messenger RNA of PKC $\epsilon$ was quantified by real-time PCR. As shown in Figure 2, $A$, PKC $\epsilon$ mRNA was significantly induced in postcardioplegic cardiac specimens ( $225 \%$ of internal control; $P<.05)$. The increased transcription of the PKC $\epsilon$ gene paralleled a 2-fold overexpression of total PKC $\epsilon$ protein $(P<.05)$, as assessed by Western blotting analysis (Figure $2, B$ ).

To verify whether the induction of the protein levels of total PKC $\epsilon$ was also associated with enhanced functional activation of this PKC isoform, protein extracts from atrial samples collected before and after cardioplegia were probed in a Western blot analysis with a specific antibody against the phosphorylated active form of PKC $\epsilon$. This blot, shown in Figure 2, $B$, documented a 2.9-fold increase in PKC $\epsilon$ phosphorylation $(P<.01)$ in the cardiac specimens exposed to cardioplegic arrest, compared with precardioplegic internal controls.

Thus, in hearts exposed to iIRI, Ucn induction at the mRNA and protein level paralleled not only a selective 


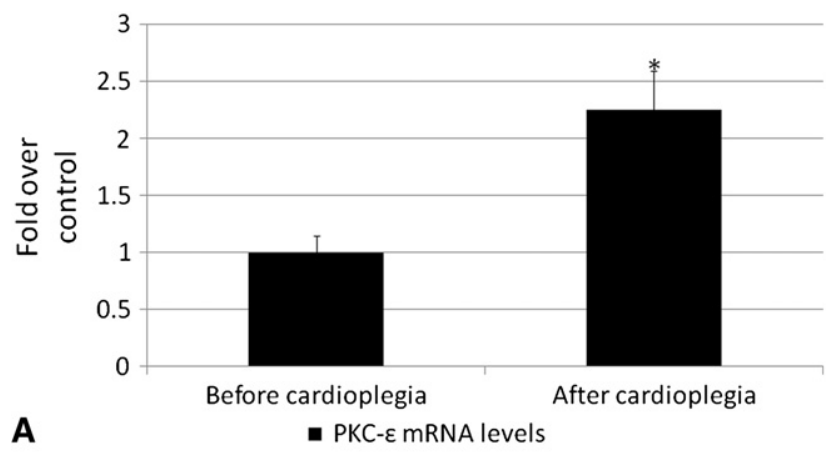

A

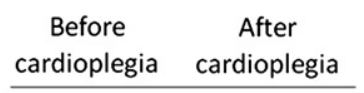

Total PKC $\varepsilon$

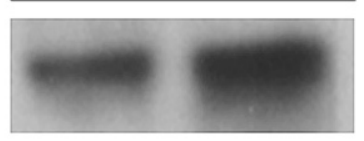

Phospho-PKC $\varepsilon$

Actin

\section{B}
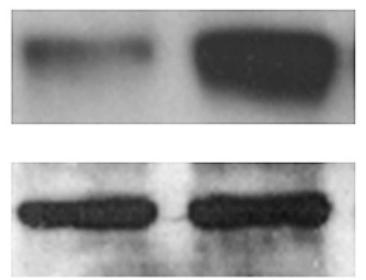

FIGURE 2. A, Quantitative PCR of PKC- $\epsilon$ mRNA levels from pre- and postcardioplegic myocardial samples. ${ }^{*} P<.05$ versus corresponding precardioplegic internal controls. B, Expression of total and phosphorylated PKC $\epsilon$ protein, as determined by Western blotting of extracts from human hearts before and after cardioplegic arrest. PKC $\epsilon$, Protein kinase $\mathrm{C} \epsilon$.

increase of PKC- $\epsilon$ mRNA and a 2-fold overexpression of total PKC $\epsilon$ isoform but also a 2.9-fold increase in PKC $\epsilon$ phosphorylation.

\section{Mitochondrial Relocation of Activated Protein \\ Kinase $\mathrm{C} \epsilon$ in Cardiac Myocytes Exposed to Cardioplegic Arrest}

To further advance our knowledge on PKC $\epsilon$-mediated cardioprotection in the human heart undergoing iIRI, we next explored whether the increased expression and activation of PKC $\epsilon$ were observed concomitantly with enhanced mitochondrial relocation. The results of this experiment are illustrated in Figure 3. Total PKC $\epsilon$ was primarily localized in the cytosolic fraction before cardioplegia, whereas

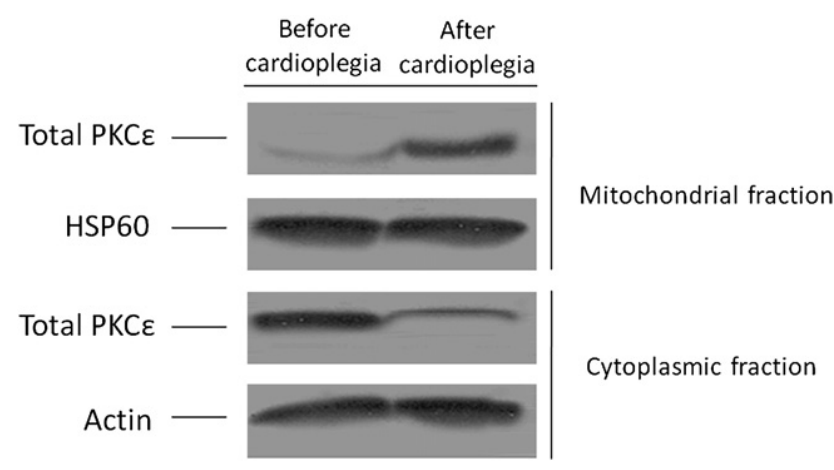

FIGURE 3. Time course of mitochondrial translocation of PKC $\epsilon$ in the human heart exposed to cardioplegic arrest as assessed by Western blotting in protein extracts from cardiac samples collected before and after cardioplegia. Hsp60 and actin antibody have been used as specific markers of the mitochondrial and cytosolic fractions, respectively. PKC $\epsilon$, Protein kinase $\mathrm{C} \epsilon$; $H s p$, heat shock protein.

after cardioplegic arrest, it was mainly detected in the mitochondrial fraction, indicating an active relocation of $\mathrm{PKC} \epsilon$ from the cytosol to the mitochondrion in the human heart undergoing iIRI $(P<.05)$.

To produce supplementary information substantiating these molecular findings, we next assessed the mitochondrial relocation of activated $\mathrm{PKC} \epsilon$ by immunohistochemistry and confocal microscopy. Numeric data and representative images pertaining to this set of experiments are presented in Table 2 and Figure 4, $A$ and $B$, respectively. In the myocardial specimens collected before cardioplegia, the intensity of the immunostaining for phosphorylated PKC $\epsilon$ was feeble and detectable in a small fraction of cardiac cells $(6.7 \% \pm$ $0.6 \%)$. In addition, in the group of cardiac cells showing this faint, although visible, positive staining for phosphorylated $\mathrm{PKC} \epsilon$, the proportion of $\mathrm{PKC} \epsilon /$ mitochondria colocalization was as low as $4.5 \% \pm 1.4 \%$ (Figure $4, A$, and Table 2). Conversely, in the biopsies collected after cardioplegic arrest, a clear positive staining for active PKC $\epsilon$ was observed in more than one fifth of cardiac myocytes $(22.4 \% \pm 3.4 \% ; P<.05)$. In this selected myocyte subpopulation, the colocalization of phosphorylated PKC $\epsilon$ and mitochondria was also dramatically increased, reaching a percentage of $56 \% \pm 8.3 \%(P<.01)$ (Figure $4, B$, and Table 2).

Thus, these results, in line with the molecular findings obtained by means of subcellular fractionation, visually

TABLE 2. Percentages of cardiac cells showing positive labeling for phosphorylated protein kinase C $\epsilon$, urocortin, and TUNEL before and after cardioplegic arrest

\begin{tabular}{lcccc}
\hline & P-PKC $\boldsymbol{\epsilon}+$ myocytes & P-PKC $\boldsymbol{\epsilon}$ /mitochondria colocalization & Urocortin & TUNEL \\
\hline Precardioplegia & $6.7 \% \pm 0.6 \%$ & $4.5 \% \pm 1.4 \%$ & $1.5 \% \pm 0.3 \%$ & $<0.1 \%$ \\
Postcardioplegia & $22.4 \pm 3.4 *$ & $56 \% \pm 8.3 \% \dagger$ & $24 \% \pm 2.9 \% \dagger$ & $3.7 \% \pm 0.6 \% *$ \\
\hline
\end{tabular}

$P$-PKC $\epsilon$, Phosphorylated protein kinase C $\epsilon$; TUNEL, terminal deoxynucleotidyl transferase-mediated nick end labeling. The proportion of colocalization between P-PKC $\epsilon$ and mitochondria in cardiac myocytes exposed or unexposed to cardioplegia is also reported. Data in percent \pm SD are expressed as the means of 12 to 15 high-power fields. $* P<.05 . \dagger P<.01$ versus corresponding precardioplegic samples. 

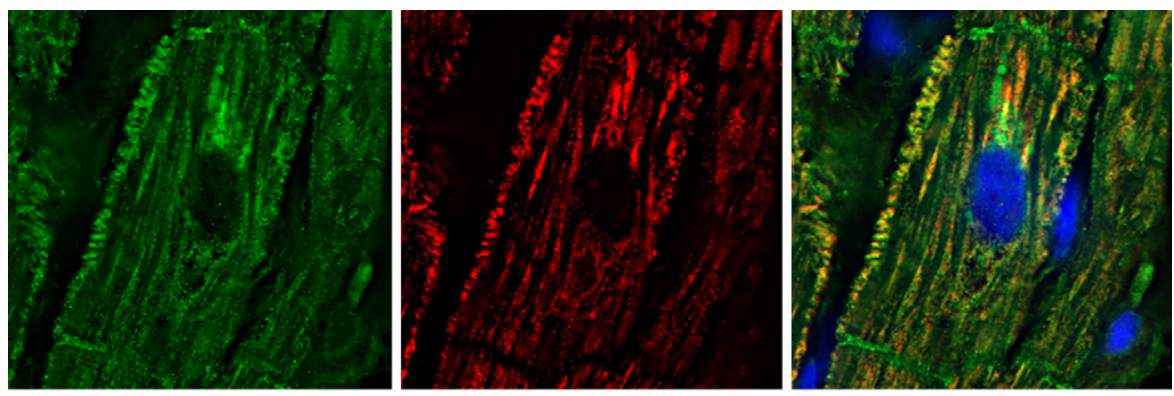

A
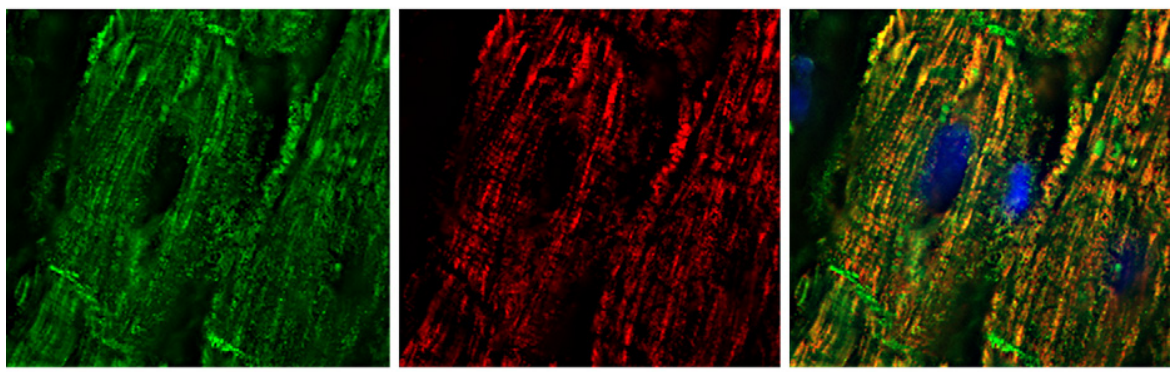

B

FIGURE 4. Myocardial sections from atrial tissues collected before (A) and after (B) cardioplegic arrest. Cardiac sections were stained with primary antibody against the phosphorylated active form of PKC $\epsilon$ and the mitochondria (Hsp60). Subsequently, the primary antibodies were labeled with secondary antibody conjugated with fluorescein (green staining in the left panels) and rhodamine (red staining in the middle panels), respectively. Finally, myocardial sections were counterstained with the nuclear labeling TOPRO-3 (blue staining in the right panels). Overlap of the 3 stainings is shown in the right panels of both figures. The percentage of colocalization of phosphorylated PKC $\epsilon$ and mitochondria was digitally quantified by means of image analyzer software. Visually, overlap of "green" phosphorylated PKC $\epsilon$ and "red" mitochondria gives rise to a bright orange fluorescent signal that can be observed in the merged images presented in the right panels.

confirmed and digitally quantified, at the cellular level, the relocation of phosphorylated PKC $\epsilon$ from the cytosolic to the mitochondrial compartment in a considerable number of cardiac cells exposed to cardioplegic arrest.

\section{Selective Mitochondrial Relocation of Protein Kinase $\mathrm{C}$ in Urocortin-Positive Myocytes Confers Protection From Apoptosis}

We next investigated whether myocytes showing mitochondrial relocation of PKC $\epsilon$ were also expressing endogenous Ucn and, if so, whether this combination was associated with protection from apoptotic cell death. To establish this correlation, 3 adjacent $5-\mu$ myocardial sections, serially cut from the same pre- and postcardioplegic wax blocks, were immunostained either for phosphorylated $\mathrm{PKC} \epsilon$ and mitochondria (Figure 5,A) or for Ucn (Figure 5, $B$ ) or TUNEL labeling (Figure 5, $C$ ). The percentage data referring to this set of experiments are reported in Table 2. Cytosolic positive staining for Ucn was detected in $1.5 \%$ $\pm 0.3 \%$ of cardiac cells before cardioplegia and in $24.6 \%$ $\pm 2.9 \%$ of the myocytes population after cardioplegic arrest $(P<.01)$. Apoptotic cell death, assessed as nuclear TUNEL positive labelling, was virtually absent in precardioplegic samples $(<0.01 \%)$ and observed in $3.7 \% \pm 0.6 \%$ of the myocyte population exposed to cardioplegic arrest $(P<.05)$. Of note, mitochondrial relocation of PKC $\epsilon$ was only observed in postcardioplegic cardiac cells with cytosolic positive staining for Ucn (Figure 5, $A-C ; P<.05$ ). Furthermore, myocytes exhibiting mitochondrial relocation of PKC $\epsilon$ and cytosolic positivity for Ucn staining at the same time were consistently TUNEL negative (Figure 5, $A-C ; P<.05$ ).

Thus, our findings show that concurrent overexpression of Ucn and mitochondrial relocation of phosphorylated PKC $\epsilon$ can be detected only in viable myocytes and that such combination is associated with protection of cardiac cells from postcardioplegic apoptotic cell death.

\section{Physical Interaction of Activated Protein Kinase C $\epsilon$ With Kir6.1 After Cardioplegic Arrest}

Having established that only cardiac cells overexpressing Ucn and showing mitochondrial relocation of activated PKC $\epsilon$ are protected from apoptosis, we investigated the mechanism by which active PKC $\epsilon$ can afford cardioprotection against apoptotic cell death, once relocated to the mitochondria. We assumed that mitochondrial PKC $\epsilon$ could physically interact with 1 or more subunits forming the mitochondrial $\mathrm{K}_{\mathrm{ATP}}$ channel. To seek validation of our postulation, we prepared additional protein extracts from tissue samples exposed or unexposed to cardioplegic arrest. Phosphorylated PKC $\epsilon$ was enriched by immunoprecipitation using a specific antibody and immunoblotted with Kir6.1, Kir6.2, or SUR-2 antibody. As shown in Figure 6, a 1.8-fold increase was 

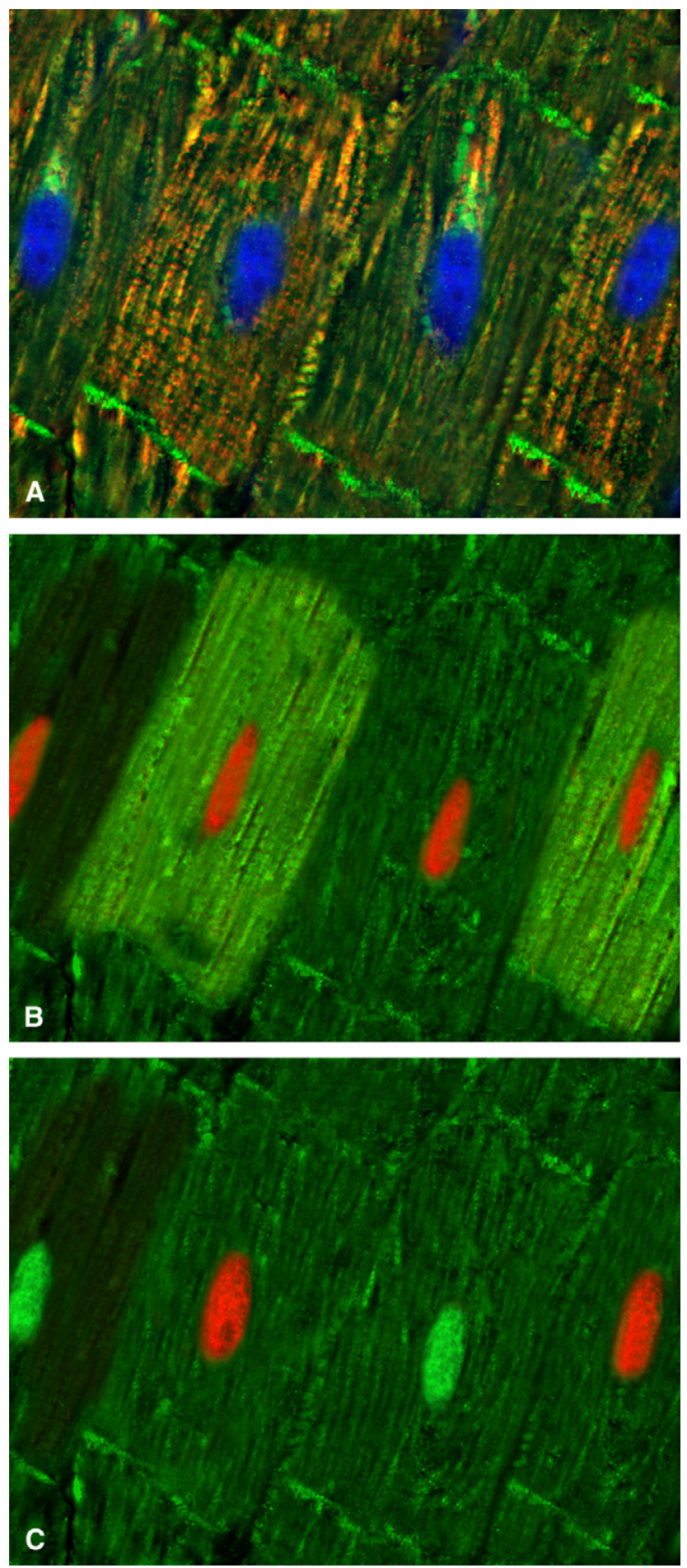

FIGURE 5. Three adjacent 5- $\mu$ myocardial sections serially cut from the same postcardioplegic wax block. The first section was stained with primary antibody against phosphorylated PKC $\epsilon$ and mitochondria (Hsp60) and counterstained with TOPRO-3 (blue nuclear staining). Secondary antibody conjugated with fluorescein or rhodamine were respectively used to label phosphorylated PKC $\epsilon$ and mitochondria. A, Overlap of the 3 stainings. B, The second section was stained with a primary antibody against Ucn, subsequently incubated with a secondary antibody conjugated with fluorescein, observed in the protein abundance of Kir6.1 in the postcardioplegic samples, compared with the internal controls $(P<.05)$, whereas no increase was observed for Kir6.2 or SUR-2. Furthermore, by immunoblotting with Kir6.1, Kir6.2, and SUR-2, the immunoprecipitation of active PKC $\epsilon$ demonstrated that phosphorylated PKC $\epsilon$ selectively interacts with the Kir6.1 subunit (although not the Kir6.2 and SUR-2 subunits) of the mitochondrial $\mathrm{K}_{\mathrm{ATP}}$ channels and that this interaction is significantly enhanced by cardioplegic arrest $(P<.05)$. Thus, our findings suggest that in the human heart exposed to cardioplegic arrest, active $\mathrm{PKC} \epsilon$, on mitochondrial relocation, physically interacts with the Kir6.1 subunit of the mitochondrial $\mathrm{K}_{\mathrm{ATP}}$ channel, and that this interaction is likely to be involved in the protection of cardiac cells against apoptotic cell death.

\section{DISCUSSION}

Apoptotic cell death has been consistently implicated in the pathogenesis of cardiac I/R injury, including the mild iatrogenic form associated with on-pump cardiac surgery. ${ }^{2-6}$ Among the several inhibitors of cardiac apoptosis reported so far, Ucn, a 40-amino acid member of the corticotropin releasing factor family, ${ }^{7}$ is especially motivating because it is endogenously expressed in the human heart, ${ }^{22}$ together with its cognate receptors. ${ }^{23}$

This report demonstrates that warm-blood cardioplegic arrest and reperfusion given to an elective population relatively comorbidity free induced enhanced expression of Ucn, at both the mRNA and the protein levels. Although previous work from our group showed postcardioplegic induction of Ucn by means of immunohistochemistry, ${ }^{7}$ the actual quantification of the Ucn protein by using a Western blotting approach had never been successful before, mainly because of the technical challenge introduced by the small molecular weight of the peptide.

In the same human heart exposed to cardioplegic arrest, Ucn overexpression was associated with increased transcription of the PKC- $\epsilon$ gene, as well as induction of the PKC- $\epsilon$ protein. This novel isoform of the PKC protein family was recently shown to play a pivotal role in the protection of the heart against $\mathrm{I} / \mathrm{R}$ injury and the mediation of ischemic preconditioning. ${ }^{14,15}$ To exert its cardioprotective effects, PKC $\epsilon$ undergoes activation by phosphorylation followed by relocation from the cytosol to the mitochondrial compartment. The importance of these 2 sequential events was substantiated by a few experimental studies, ${ }^{24}$ but, to the best of our knowledge, was never investigated in the clinical setting. Our study shows that in the hearts of patients with

and finally counterstained with propidium iodide (red nuclear staining). C, The third section was stained with TUNEL reagents and counterstained with propidium iodide (red nuclear labeling). TUNEL-positive nuclei are stained bright green. 


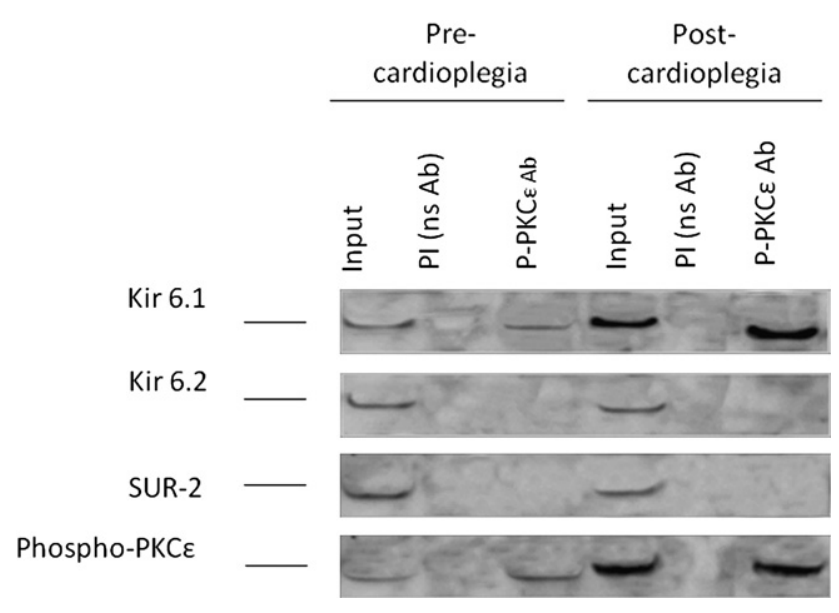

FIGURE 6. Co-immunoprecipitation of phosphorylated PKC $\epsilon$ with Kir6.1, Kir6.2, and SUR-2 in protein extracts from pre- and postcardioplegic cardiac biopsies. Phosphorylated PKC $\epsilon$ was enriched by immunoprecipitation using a specific antibody and immunoblotted with Kir6.1, Kir6.2, or SUR-2 antibody. Input: protein extracts. PI, Pre-immune control antibody; $P$-PKC€ $A b$, immunoprecipitation antibody against phosphorylated PKC $\epsilon$; $P K C \epsilon$, protein kinase $\mathrm{C} \epsilon$.

minimal comorbidities undergoing warm-blood cardioplegic arrest, the induction of PKC $\epsilon$, at both the mRNA and the protein levels, paralleled enhanced activation and mitochondrial relocation of this PKC isoform. Cardiac myocytes exhibiting at the same time mitochondrial relocation of $\mathrm{PKC} \epsilon$ and cytosolic positive staining for Ucn were all viable, being consistently TUNEL-negative. Overexpression of Ucn together with PKC $\epsilon$ relocation and cell survival have been systematically observed in all low-risk patients of our elective population (whose surgical procedure and postsurgical follow-up were uneventful) and may represent the ideal response of the human heart to cardioplegic arrest in the absence of comorbidities. We are currently investigating whether diversities in cardiac response to cardioplegia can provide a molecular explanation for the poor postsurgical outcomes observed in patients with comorbidities, such as diabetes mellitus.

Recent studies have reported that exogenous Ucn can induce activation and mitochondrial translocation of PKC $\epsilon .{ }^{15}$ In addition, pharmacologic inhibition of $\mathrm{PKC} \epsilon$, or selective knockout of its gene, was shown to abrogate Ucn-mediated cardioprotection. ${ }^{15}$ Although intriguing, our data in the postcardioplegic human heart are mainly conjectural and cannot prove such a causative link among Ucn expression, PKC $\epsilon$ activation/relocation, and cell survival. To prove that Ucn-mediated cardioprotection is dependent on PKC $\epsilon$ activation and mitochondrial translocation, a doubleblinded, randomized clinical trial evaluating the cardioprotective effects of exogenous Ucn prophylactically infused to patients undergoing cardioplegic arrest is currently under way at the University of Verona, Italy.
Several pieces of evidence have also documented that PKC $\epsilon$, once activated and translocated to the mitochondria, can interact with different mitochondrial proteins. ${ }^{25,26}$ Once again, this multitude of experimental data stand alone, being unmatched in the human heart. We show that in patients undergoing cardioplegic arrest, activated PKC $\epsilon$ translocates to the mitochondria, where it physically interacts with the Kir6.1 subunit of the mitochondrial $\mathrm{K}_{\mathrm{ATP}}$ channel. This interaction is selective because it was not observed for the other 2 components of the channels, represented by Kir6.2 and SUR-2. Because the cardioprotective effects of Ucn, in the isolated cardiac cells and the intact heart, are specifically blocked by both pharmacologic and genetic inhibition of PKC $\epsilon,{ }^{15}$ as well as by generalized and mitochondrial-specific $\mathrm{K}_{\mathrm{ATP}}$ channel blockers, ${ }^{11}$ we may postulate that one mechanism of Ucn-mediated cardioprotection involves induction and activation of PKC $\epsilon$, followed by $\mathrm{PKC} \epsilon$ mitochondrial translocation and interaction with the Kir6.1 subunit, finally resulting in opening of the mitochondrial $\mathrm{K}_{\mathrm{ATP}}$ channel. Although the data we present in this article do not enable mechanistic conclusions, similar considerations on the mechanism of protection of Ucn may also apply to the human heart exposed to iIRI.

\section{CONCLUSIONS}

We showed that cardioplegic arrest induces overexpression of endogenous Ucn, at both the mRNA and the protein levels, in elective low-risk patients undergoing on-pump cardiac surgery. This induction colocalized with PKC $\epsilon$ activation, mitochondrial translocation, and myocyte survival against apoptotic cell death. Because the loss and functional impairment of myocytes after cardioplegic arrest are known to directly reduce cardiac contractility, resulting in greater mortality and morbidity associated with on-pump cardiac surgery, ${ }^{3,4}$ interventions aimed at reducing the extent of apoptotic cell death postoperatively are warranted. Among the several strategies proposed, supplementation of cardioplegic solutions with exogenous Ucn seems to be promising to reduce the risk of postsurgical cardiac dysfunction in patients exposed to the inescapable $\mathrm{I} / \mathrm{R}$ injury associated with CABG.

\section{References}

1. Mauney MC, Kron IL. The physiologic basis of warm cardioplegia. Ann Thorac Surg. 1995;60:819-23.

2. Schmitt JP, Schröder J, Schunkert H, Birnbaum DE, Aebert H. Role of apoptosis in myocardial stunning after open heart surgery. Ann Thorac Surg. 2002;73: 1229-35.

3. Chen YF, Wu SC, Huang CH, Pan PC, Lee CS, Lin CC. Morphometric identification of luminal narrowing of myocardial capillaries after cardioplegic arrest. Ann Thorac Surg. 2001;71:243-8.

4. Rainio P, Kaukoranta PK, Sormunen R, Juvonen T, Peuhkurinen KJ. Ultrastructural changes in myocardium during mild hypothermic retrograde blood cardioplegia. Scand Cardiovasc J. 1998;32:353-9.

5. Aebert H, Kirchner S, Keyser A, et al. Endothelial apoptosis is induced by serum of patients after cardiopulmonary bypass. Eur J Cardiothorac Surg. 2000;18: 589-93. 
6. Ramlawi B, Feng J, Mieno S, et al. Indices of apoptosis activation after blood cardioplegia and cardiopulmonary bypass. Circulation. 2006;114(1 Suppl): I257-63.

7. Scarabelli TM, Pasini E, Ferrari G, et al. Warm blood cardioplegic arrest induces mitochondrial-mediated cardiomyocyte apoptosis associated with increased urocortin expression in viable cells. J Thorac Cardiovasc Surg. 2004;128:364-71.

8. Lawrence KM, Chanalaris A, Scarabelli T, et al. K(ATP) channel gene expression is induced by urocortin and mediates its cardioprotective effect. Circulation. 2002; 106:1556-62.

9. Noma A. ATP-regulated K+channels in cardiac muscle. Nature. 1983;305:147-8.

10. Cole WC, McPherson CD, Sontag D. ATP-regulated K+ channels protect the myocardium against ischemia/reperfusion damage. Circ Res. 1991;69:571-81.

11. O'Rourke B. Myocardial K(ATP) channels in preconditioning. Circ Res. 2000;87: 845-55.

12. Costa AD, Garlid KD, West IC, et al. Protein kinase G transmits the cardioprotective signal from cytosol to mitochondria. Circ Res. 2005;97:329-36.

13. Murriel CL, Mochly-Rosen D. Opposing roles of delta and epsilon PKC in cardiac ischemia and reperfusion: targeting the apoptotic machinery. Arch Biochem Biophys. 2003;420:246-54.

14. Liu GS, Cohen MV, Mochly-Rosen D, Downey JM. Protein kinase C-epsilon is responsible for the protection of preconditioning in rabbit cardiomyocytes. $J \mathrm{Mol}$ Cell Cardiol. 1999;31:1937-48.

15. Lawrence KM, Kabir AM, Bellahcene M, et al. Cardioprotection mediated by urocortin is dependent on PKCepsilon activation. FASEB J. 2005;19:831-3.

16. Tichopad A, Dilger M, Schwarz G, Pfaffl MW. Standardized determination of realtime PCR efficiency from a single reaction set-up. Nucleic Acids Res. 2003;31:e122.

17. Schägger H. Tricine-SDS-PAGE. Nat Protoc. 2006;1:16-22.
18. Scarabelli TM, Stephanou A, Pasini E. Different signaling pathways induce apoptosis in endothelial cells and cardiac myocytes during ischemia/reperfusion injury. Circ Res. 2002;90:745-8.

19. Scarabelli TM, Stephanou A, Pasini E, et al. Minocycline inhibits caspase activation and reactivation, increases the ratio of XIAP to smac/DIABLO, and reduces the mitochondrial leakage of cytochrome $\mathrm{C}$ and smac/DIABLO. J Am Coll Cardiol. 2004;43:865-74.

20. Townsend PA, Cutress RI, Carroll CJ, et al. BAG-1 proteins protect cardiac myocytes from simulated ischemia/reperfusion-induced apoptosis via an alternate mechanism of cell survival independent of the proteasome. J Biol Chem. 2004 279:20723-8.

21. Scarabelli T, Stephanou A, Rayment N, et al. Apoptosis of endothelial cells precedes myocyte cell apoptosis in ischemia/reperfusion injury. Circulation. 2001; 104:253-6.

22. Nishikimi T, Miyata A, Horio T, et al. Urocortin, a member of the corticotropinreleasing factor family, in normal and diseased heart. Am J Physiol Heart Circ Physiol. 2000;279:H3031-9.

23. Chen R, Lewis KA, Perrin MH, Vale WW. Expression cloning of a human corticotropin-releasing-factor receptor. Proc Natl Acad Sci U S A. 1993;90:8967-71.

24. Inagaki K, Churchill E, Mochly-Rosen D. Epsilon protein kinase $\mathrm{C}$ as a potential therapeutic target for the ischemic heart. Cardiovasc Res. 2006;70:222-30.

25. Ohnuma Y, Miura T, Miki T, et al. Opening of mitochondrial K(ATP) channel occurs downstream of PKC-epsilon activation in the mechanism of preconditioning. Am J Physiol Heart Circ Physiol. 2002;283:H440-7.

26. Baines CP, Song CX, Zheng YT, et al. Protein kinase $\mathrm{C}$ epsilon interacts with and inhibits the permeability transition pore in cardiac mitochondria. Circ Res. 2003; 92:873-80. 